\title{
A Novel Biochip Detection System Based on Lateral SPR Wave Propagation Theory
}

\author{
Lin, Chii-Wann, Song, Zhi-Hsiang, Lee, Chih-Kung \\ Institute of Biomedical Engineering \& Department of Electronics Engineering, \\ National Taiwan University, Taipei, ROC, cwlinx@ntu.edu.tw \\ Institute of Biomedical Engineering, National Taiwan University, Taipei, Taiwan, ROC \\ Institute of Applied Mechanics, National Taiwan University, Taipei, Taiwan, ROC, cklee@ntu.edu.tw
}

\begin{abstract}
In this paper, we investigated the lateral SPR propagation theory and implemented a novel optical SPR detection system, using two-prism coupling method to excite SPR and detecting the lateral propagating SEW, which by enlarging the sensing area of SPR thus enhance the detection sensitivity. From theoretical calculation, the propagation length is increasing with excitation wavelength and provides the detection sensitivity $\sim 1.05 * 10^{-5}$ RIU at $\lambda=790 \mathrm{~nm}$. By measuring the propagation length (at fixed wavelength) or the resonant wavelength (at fixed lateral length) changes around SPR, we can use this lateral SPR phenomenon for biochip applications.
\end{abstract}

\section{Keywords}

Surface Plasmon Resonance, Lateral propagation, Biochip.

\section{INTRODUCTION}

The uniqueness of biosensor is the integration of biological elements as a part of the sensing mechanism for recognition process and provides analytical information of the specific components or total compositions. With the enabling technologies of semiconductors and Micro Electro-Mechanical System (MEMS), biochip offers massive parallelism and possible automation for high throughput. Among the available sensing mechanisms, optical methods are widely used in many biochip systems. Surface plasmon resonance (SPR) sensor has been one of the focal technologies due to its non-labeling and real-time merits. It is highly sensitive to the near surface refractive index changes, which might be due to local composition and/or concentration variations, and results in shifting of resonant position, wavelength. SPR can be induced and propagated along a metallic and dielectric interface by coupling proper EM-wave excitation. Its maximal electrical field due to forced oscillation of applied TM wave exhibits exponential decay on both parallel and perpendicular directions as evanescent waves or surface plasmon wave (SPW). The majority of the available SPR sensors are based on field extension of z-direction to probe the interaction with molecules. We will shift our focus to the ignored aspect of SPW along x-direction or lateral propagation for possible bio-applications. As a matter of fact, this phenomenon, especially its propagation length, had been studied in the past. For examples, Schoenwald reported a two-prism coupling method to excite SPW and measure its propagation distance by decoupling from the second prism [7]. There were also reports from many other groups that SPW can sustain quite a long distance on various metallic surfaces mainly with infrared light source or radio frequency EM waves [5, 2, 3, 1, 8]. It can be used to probe the thin film properties on a metallic surface along its propagating distance. The combination of two-prism coupling, multiple total internal reflection (MTIR) and infrared absorption spectrum (IRAS) resulted in a ultra-sensitive optical analytical technology for the identification of residual organic contaminations on silicon wafer during fabrication processes [6]. To our knowledge, this technology has not been applied as a sensing mechanism for biosensor yet. The purposes of this paper will first investigate the lateral propagation theory of SPW and then report the implementation of a novel optical SPR detection system, using twoprism coupling method

\section{MATERIALS AND METHODS}

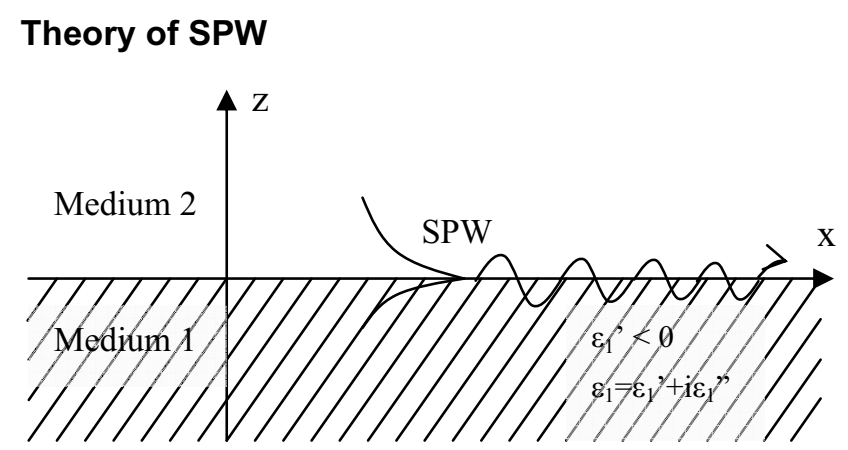

Figure 1. SEW on the interface between two medium of dielectric function of $\varepsilon_{1}, \varepsilon_{2}$

As shown in the Figure 1, a TM-polarized light into medium $2\left(\varepsilon_{2}=\varepsilon^{\prime}{ }_{2}+i \varepsilon{ }_{2}\right)$ and medium $1\left(\varepsilon_{1}=\varepsilon^{\prime}{ }_{1}+i \varepsilon{ }_{1}\right)$, the solutions for such a SPW can be derived from Maxwell equations with proper boundary conditions. We can get equations for $k_{x}, k_{z 1}$, and $k_{z 2}$ as follows: 


$$
\begin{aligned}
& k_{x}^{2}=\left(\frac{\varepsilon_{1} \varepsilon_{2}}{\varepsilon_{1}+\varepsilon_{2}}\right)\left(\frac{\omega}{c}\right)^{2} \rightarrow \text { Dispersion relation of surface plasmon } \\
& k_{z 1}^{2}=\left(\frac{\varepsilon_{1}^{2}}{\varepsilon_{1}+\varepsilon_{2}}\right)\left(\frac{\omega}{c}\right)^{2} \\
& k_{z 2}^{2}=\left(\frac{\varepsilon_{2}^{2}}{\varepsilon_{1}^{2}+\varepsilon_{2}^{2}}\right)\left(\frac{\omega}{c}\right)^{2}
\end{aligned}
$$

With the assumptions of non-absorbing material $\left(\varepsilon_{2}=\varepsilon^{\prime}{ }_{2}\right)$ and $\varepsilon{ }^{\prime}{ }_{1}<\left|\varepsilon{ }_{1}\right|$, one can get the reduced form as follows:

$$
\begin{aligned}
& k_{x}^{2}=\left(\frac{\omega}{c}\right)^{2}\left(\frac{\left(\varepsilon_{1}^{\prime} \varepsilon_{2}^{\prime}\left(\varepsilon_{1}^{\prime}+\varepsilon_{2}^{\prime}\right)+\varepsilon_{1}^{\prime 2} \varepsilon_{2}^{\prime}\right)+i\left(\varepsilon_{1}^{\prime \prime} \varepsilon_{2}^{\prime 2}\right)}{\left(\left(\varepsilon_{1}^{\prime}+\varepsilon_{2}^{\prime}\right)^{2}+\left(\varepsilon_{1}^{\prime \prime}\right)^{2}\right)}\right) \\
& \operatorname{Re}\left(k_{x}\right)=\frac{\omega}{c}\left(\frac{\varepsilon_{1}^{\prime} \varepsilon_{2}}{\varepsilon_{1}^{\prime}+\varepsilon_{2}}\right)^{1 / 2} \\
& \operatorname{Im}\left(k_{x}\right)=\frac{\omega}{c}\left(\frac{\varepsilon_{1}^{\prime} \varepsilon_{2}}{\varepsilon_{1}^{\prime}+\varepsilon_{2}}\right)^{3 / 2} \frac{\varepsilon_{1}^{\prime \prime}}{2 \varepsilon_{1}^{\prime 2}}
\end{aligned}
$$

For the real part of $k_{x}$, one can use metal or doped semiconductors to fulfill $\varepsilon^{\prime}{ }_{1}<0$ and $\left|\varepsilon^{\prime_{1}}\right|>\varepsilon_{2}$ requirements. The lateral propagation of SPW is the exponential decay of its "energy intensity", which is proportional to the square of electrical field, along the x-direction as shown in the Figure 1. The propagation length $\left(L_{x}\right)$ can be defined as the characteristic distance of maximum resonant energy drops to its $1 / e\left(I=I_{0} / e\right)$. It depends on the $\operatorname{Im}\left(k_{x}\right)$ and its internal absorption, where:

$$
L x=\left[2 \operatorname{Im}\left(k_{x}\right)\right]^{-1}=\frac{c}{\omega}\left(\frac{\varepsilon_{1}^{\prime}+\varepsilon_{2}}{\varepsilon_{1}^{\prime} \varepsilon_{2}}\right)^{-3 / 2} \frac{2 \varepsilon_{1}^{\prime 2}}{\varepsilon_{1}^{\prime \prime}}
$$

As lateral propagation is based on the perturbation of electrons, $L_{x}$ is also related to dc conductivity of metals and can be expressed as follow:

$$
L_{x}=\sigma_{d c} / 2 \pi^{2} c \varepsilon_{\infty} \omega^{2}=15 \lambda^{2} / \pi^{2} \varepsilon_{\infty} \rho
$$

\section{Chip Design and Fabrication}

There are several available configurations can be used to excite SPR, i.e. Otto, Kretschmann, and long range. However, Otto type with proper control of air gap or the thickness of dielectric layer is the best design possibly due to its coupling into waveguide mode. We adapted this configuration in this paper as well. However, the control of gap height is an important parameter for the performance of lateral propagation. As a general rule of thumb, the air gap should be about one half of the field penetration depth in air with specified wavelength. For example, the near optimal design for the use of such a sensor in air (air / $\mathrm{Au} /$ glass $)$ or water $\left(\mathrm{H}_{2} \mathrm{O} / \mathrm{Au} /\right.$ glass $)$ at $633 \mathrm{~nm}$, the required minimal $\mathrm{Au}$ film thickness is $200 \mathrm{~nm}$ and the gap is about 400 500nm. As shown in the Figure 2, our design used 200

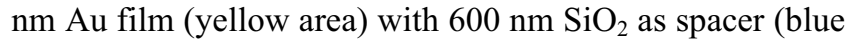
area) to have a gap height of $400 \mathrm{~nm}$.
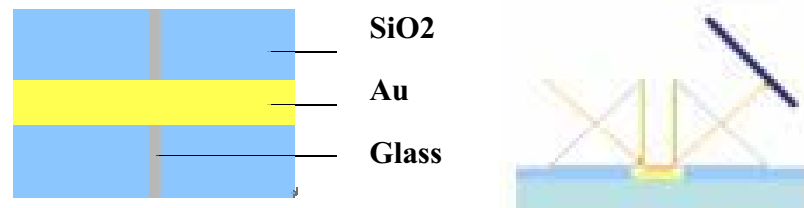

Figure 2 The design of SPR chip for later propagation and device setup. Measuring steps of lateral SPR, from $x=0$ to $x=L x$ (defined as the distance where reflection light intensity decays to $1 / \mathrm{e})$.

\section{Fabrication}

Glass slides were cleaned with piranha solution, and rinsed with Nanopure water, and finally rinsed with ethanol. The $\mathrm{Au}$ film was deposited by evaporation of $\sim 1 \mathrm{~nm}$ of $\mathrm{Cr}$ followed by deposition of $\mathrm{Au}$ at a pressure of $\sim 1.6^{*} 10^{-6}$ torr using an E-beam evaporator at the Precision Instrument Development Center (PIDC, Hsin-Chu). The deposition rate was $0.1-0.2 \mathrm{~nm} / \mathrm{s}$. The thickness of the chromium and gold were monitored by a quartz crystal microbalance, which has been carefully calibrated according to the different characteristics of deposited materials.

\section{Measurement Setup}

To measure the changes caused by samples, we can use the optical system setup as shown in the Figure 3 to measure the intensity changes with specific $L_{x}$. One can use the $\Delta L_{x}$ to calculate the $\Delta n$ on the metallic surface.

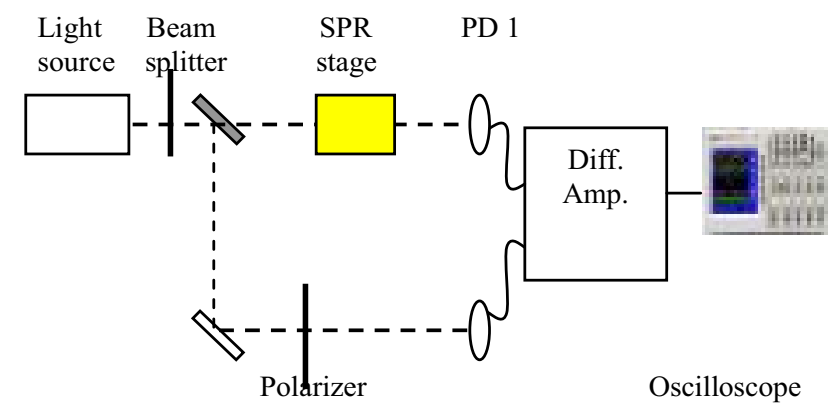

Figure 3. Block diagram of optical system setup.

\section{RESULTS AND DISCUSSIONS}

\section{Theoretical Simulation}

According to the theoretical derivation, we can plot the propagation length and penetration depth on $\mathrm{x}$ and $\mathrm{z}$ direction. Figure 4 shows the 3D plot of $x$ and $z$ direction field intensity at SPR angle with $633 \mathrm{~nm}$ incident light and $\mathrm{Au}$ film in the water. The propagation length of Lx is about 3.3 um according to the definition in this example case. Table 1 shows the summary of wavelength effects on gap height, SPR angle and Lx. We can then calculate the effect of medium's refractive index on $L_{x}$ and its sensitivity $\left(d L_{x} / d n\right)$ with different wavelengths as shown in the Figure 5. 


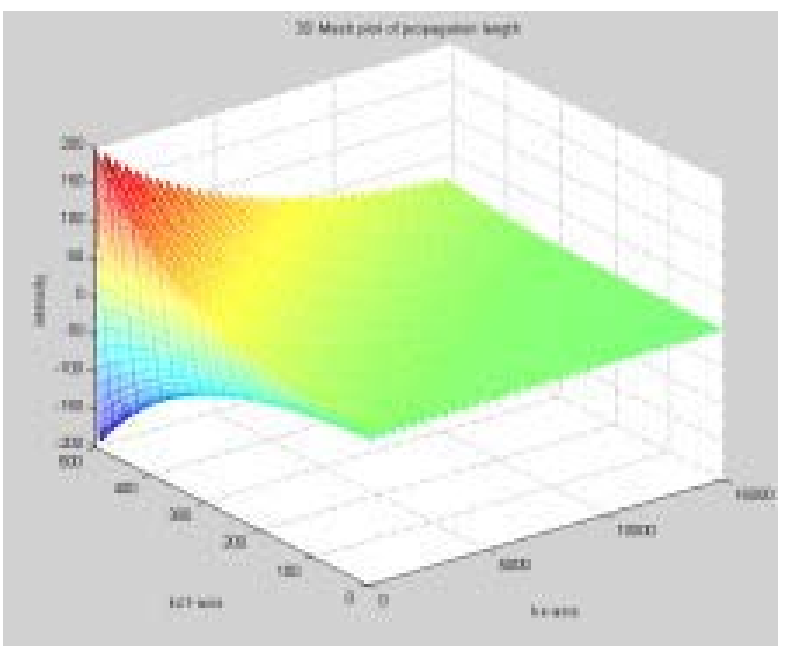

Figure 4. 3D plot of $\mathrm{x}$ and $\mathrm{z}$ direction (in $\mathrm{nm}$ units) field intensity with $\lambda=633 \mathrm{~nm}$ and $A u$ film in the water.
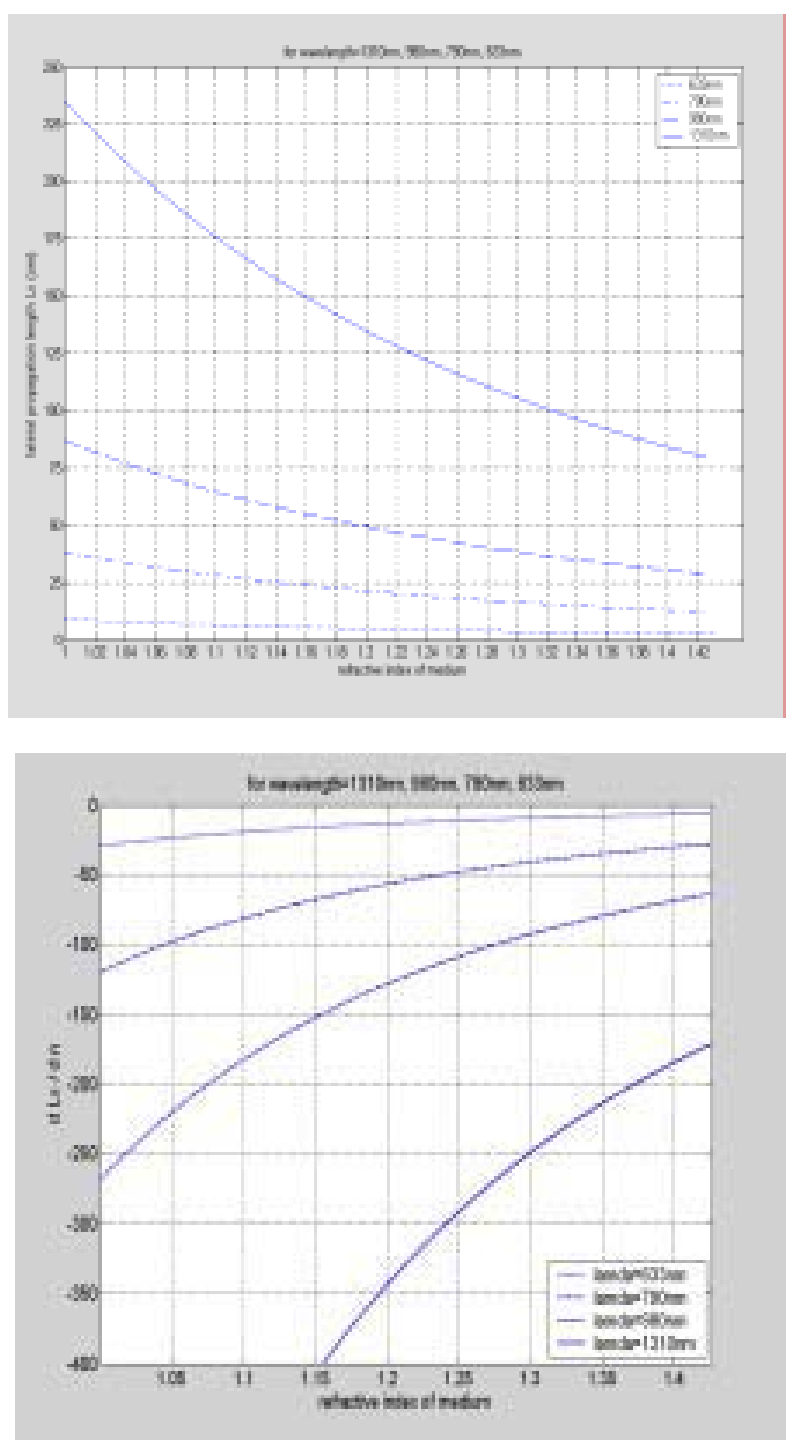

Figure 5. Theoretical Simulation of the (a) propagation length and (b) sensitivity, dLx/dn due to four different wavelength $\left(L_{x}=\left(\varepsilon_{1}^{2}+\varepsilon_{2}^{2}\right) / 2 \pi \eta_{1}^{3 / 2} \varepsilon_{2} \omega\right)$.
Table 1. Summary of different wavelength effects on gap height, SPR angle and Lx.

\begin{tabular}{|c|c|c|c|c|}
\hline \multicolumn{5}{|c|}{ Au film } \\
\hline wavelength & $633 \mathrm{~nm}$ & $790 \mathrm{~nm}$ & $980 \mathrm{~nm}$ & $1310 \mathrm{~nm}$ \\
\hline Air gap & $550 \mathrm{~nm}$ & $1.1 \mu \mathrm{m}$ & $1.8 \mu \mathrm{m}$ & $3 \mu \mathrm{m}$ \\
\hline SPR angle & $43.85^{\circ}$ & $42.6^{\circ}$ & $42.24^{\circ}$ & $41.97^{\circ}$ \\
\hline $\mathrm{L}_{\mathrm{x}}$ (um) & 8.88 & 38.125 & 86.912 & 94.328 \\
\hline H2O gap & $260 \mathrm{~nm}$ & $620 \mathrm{~nm}$ & lum & $1.8 \mathrm{um}$ \\
\hline SPR angle & $77.2^{\circ}$ & $66.45^{\circ}$ & $64^{\circ}$ & $62.15^{\circ}$ \\
\hline $\mathrm{L}_{\mathrm{x}}$ (um) & 3.30 & 15.417 & 36.381 & 99.751 \\
\hline
\end{tabular}

In short, the effects of longer incident wavelength include:

- Refractive index of water will decrease;

- Decreasing in real part and increasing in imaginary part of dielectric function for $\mathrm{Au}$;

- Increasing of $\mathrm{Lx}$ in both air/Au and $\mathrm{H} 2 \mathrm{O} / \mathrm{Au}$;

- Decreasing in penetration depth within Au;

- Increasing in penetration depth within dielectric material.

\section{Fabricated Device and Measurement}

Figure 6 shows the fabricated devices according to above mentioned design of various widths for different sizes of coupling prisms, which have a reflective coating on one side to avoid stray scattering effect on SPR coupling.
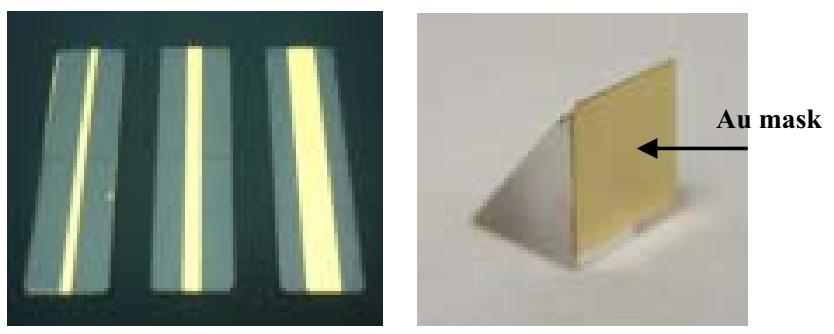

Figure 6 The fabricated SPR devices and coupling prism with Au mask on one side to avoid possible scattering effect due to stray light.

Lateral propagation of SPW can be measured by using a CCD camera with similar setup as mentioned above to image intensity change while angle changes as shown in the Figure 7. It clearly demonstrates SPW reaching its maximal propagation length near the SPR angle with $\mathrm{SF} 10 / \mathrm{H} 2 \mathrm{O} / \mathrm{Au}$. We then looked at the intensity changes with different distance between two prisms for 5 different incident angles at $633 \mathrm{~nm}$. As shown in Figure 8, we can not only find the reflective intensity reaching its minimum at 43.2 degree but also its curve fitting approaching theoretical calculation with $\mathrm{y}=\mathrm{A} 1 * \exp (-\mathrm{x} / \mathrm{t} 1)+\mathrm{y} 0$, where $\mathrm{y}$ is the reflective intensity, $\mathrm{t} 1$ is the relaxation time, and $\mathrm{x}$ is the distance in um. It was found $\mathrm{t} 1$ is $2.85 \times 10^{-6}$ in this case. The theoretical calculation of exponential decay $\left(\exp \left(-2 \mathrm{k}_{\mathrm{x}}{ }^{\prime} \mathrm{x}\right)\right)$ gives $2 \mathrm{k}_{\mathrm{x}}{ }^{\prime \prime}=1.14 * 10^{5}$, at $43.2 \mathrm{deg}$. 

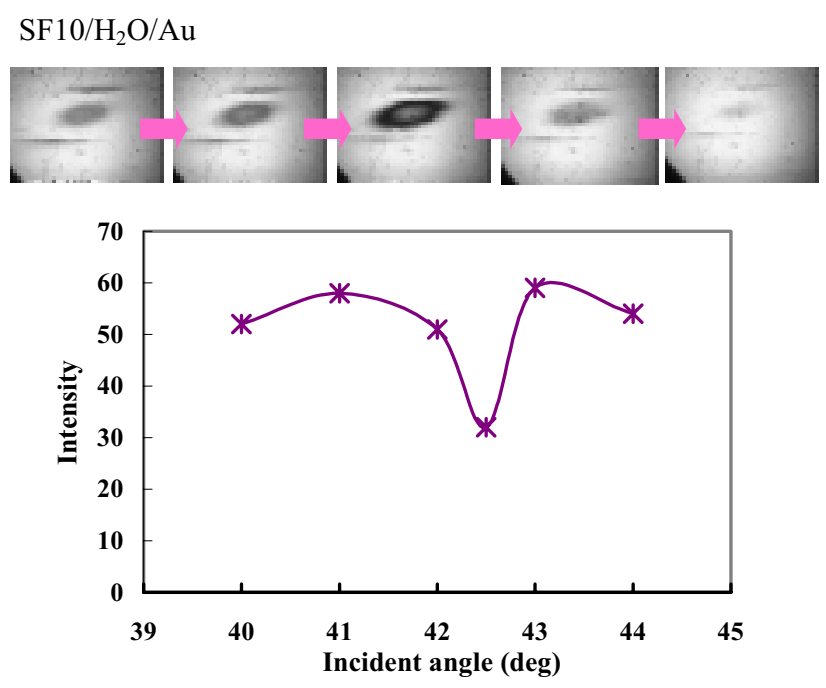

Figure 7. Lateral SPW experiment result-- increasing distance between two prisms vs. reflection intensity at different incident angle of $\mathrm{Au} / \mathrm{air}$ and $\mathrm{Au} / \mathrm{H} 2 \mathrm{O}$. The lateral length increases to its maximum when the incident angle near SPR angle.

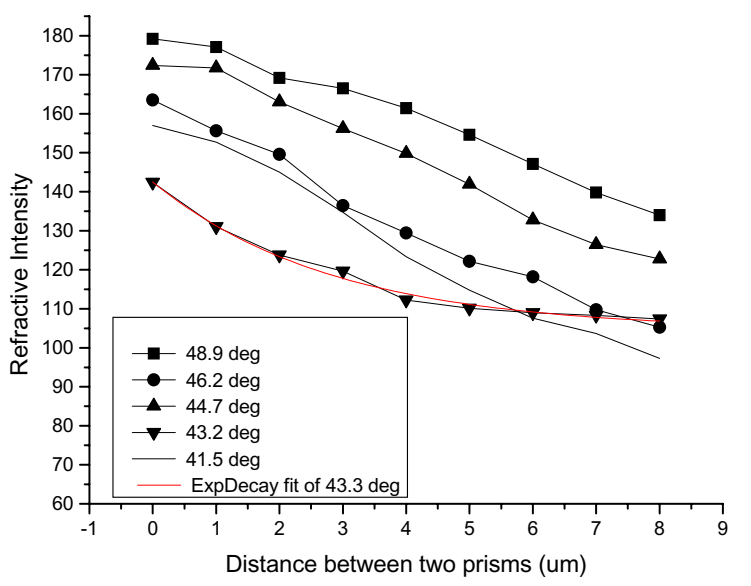

Figure 8. Reflective intensity changes due to the modulation of distance between two coupling prisms with 5 different incident angles. The fitting of $\mathbf{4 3 . 2}$ degree to an exponential decay function of $y=A 1 * \exp (-x / t 1)+y 0$ and compared to theoretical $\operatorname{model}$ of $\exp \left(-2 k_{x}{ }^{\prime} x\right)$ for the values of $1 / t 1$ and $k_{x}$ ".

\section{CONCLUSIONS}

Lateral SPW propagation theory and its implementation using two-prism coupling method have been demonstrated in this paper. From theoretical calculation, the propagation length is increasing with excitation wavelength and provides the detection sensitivity $\sim 1.05^{*} 10^{-5}$ RIU at $\lambda=790 \mathrm{~nm}$. By measuring the propagation length (at fixed wavelength) or the resonant wavelength (at fixed lateral length) changes around SPR, we can use this lateral SPR phenomenon for biochip applications.

\section{ACKNOWLEDGMENTS}

This project is supported in part by National Science Council, Minister of Economic Affairs of Taiwan, Republic of China.

\section{REFERENCES}

[1] R.W. Alexander, R.J. Bell, C.A. Ward, "Applications of Surface Electromagnetic Waves to Measure Absorption Coefficients", J. Chem. Phys. Vol: 59, p.3492. (1973)

[2] A.S. Barker, "Optical Measurements of Surface Plasmons in Gold", Phys. Rev. Vol: B8, p.5418 (1973)

[3] R.J. Bell, R.W. Alexander, Jr., W.F. Parks, G. Kovener, I.L. Tyler, "Surface excitations in absorbing media", Opt. Commun. Vol: 8, p.147 (1973)

[4] R.J. Bell, R.W. Alexander, Jr., C.A. Ward, I.L. Tyler, "Introductory theory for surface electromagnetic wave spectroscopy", Surface Science Vol: 48, p.253 (1975)

[5] G. Goubau, "Surfacewaves and their applications to transmission lines", J. Appl. Phys. Vol: 21, p.1119. (1950)

[6] M. Olivier, N. Rochat, A. Chabli, G. Lefeuvre, F. Conne, "Infrared Study of Hydrogen in Ultra-Thin Silicon Nitride Films Using Multiple Internal Reflection Spectroscopy (MIR) in $200 \mathrm{~mm}$ Silicon Wafers", phys. stat. sol. (a) Vol: 175, p.137 (1999)

[7] J. Schoenwald, E. Burstein, M. Elson, "Propagation of surface polaritons over macroscopic distances at optical frequencies", Solid State Comm. Vol: 12, p.185. (1973)

[8] C.A. Ward, R.J. Bell, R.W. Alexander, G. Kovener, "Surface electromagnetic waves on metals and polar insulators: some comments", Appl. Opt. Vol: 13, p.2378. (1974) 